\title{
Refining the predictive variables in the "Surgical Risk Preoperative Assessment System" (SURPAS): a descriptive analysis
}

William G. Henderson 1,2,3, Michael R. Bronsert ${ }^{1,2}$, Karl E. Hammermeister ${ }^{1,2,4}$, Anne Lambert-Kerzner ${ }^{1,2,5}$ and Robert A. Meguid ${ }^{1,2,6^{*}}$ (D)

\begin{abstract}
Background: The Surgical Risk Preoperative Assessment System (SURPAS) is a parsimonious set of models providing accurate preoperative prediction of common adverse outcomes for individual patients. However, focus groups with surgeons and patients have developed a list of questions about and recommendations for how to further improve SURPAS's usability and usefulness. Eight issues were systematically evaluated to improve SURPAS.

Methods: The eight issues were divided into three groups: concerns to be addressed through further analysis of the database; addition of features to the SURPAS tool; and the collection of additional outcomes. Standard multiple logistic regression analysis was performed using the 2005-2015 American College of Surgeons National Surgical Quality Improvement Participant Use File (ACS NSQIP PUF) to refine models: substitution of the preoperative sepsis variable with a procedure-related risk variable; testing of an indicator variable for multiple concurrent procedure codes in complex operations; and addition of outcomes to increase clinical applicability. Automated risk documentation in the electronic health record and a patient handout and supporting documentation were developed. Long term functional outcomes were considered.
\end{abstract}

Results: Model discrimination and calibration improved when preoperative sepsis was replaced with a procedure-related risk variable. Addition of an indicator variable for multiple concurrent procedures did not significantly improve the models. Models were developed for a revised set of eleven adverse postoperative outcomes that separated bleeding/ transfusion from the cardiac outcomes, UTI from the other infection outcomes, and added a predictive model for unplanned readmission. Automated documentation of risk assessment in the electronic health record, visual displays of risk for providers and patients and an "About" section describing the development of the tool were developed and implemented. Long term functional outcomes were considered to be beyond the scope of the current SURPAS tool.

Conclusion: Refinements to SURPAS were successful in improving the accuracy of the models, while reducing manual entry to five of the eight variables. Adding a predictor variable to indicate a complex operation with multiple current procedure codes did not improve the accuracy of the models. We developed graphical displays of risk for providers and patients, including a take-home handout and automated documentation of risk in the electronic health record. These improvements should facilitate easier implementation of SURPAS.

\footnotetext{
* Correspondence: ROBERT.MEGUID@UCDenver.edu

'Surgical Outcomes and Applied Research program, Department of Surgery,

University of Colorado School of Medicine, Aurora, CO, USA

${ }^{2}$ Adult and Child Center for Health Outcomes Research and Delivery Science,

University of Colorado School of Medicine, Aurora, CO, USA

Full list of author information is available at the end of the article
}

(c) The Author(s). 2019 Open Access This article is distributed under the terms of the Creative Commons Attribution 4.0 International License (http://creativecommons.org/licenses/by/4.0/), which permits unrestricted use, distribution, and reproduction in any medium, provided you give appropriate credit to the original author(s) and the source, provide a link to the Creative Commons license, and indicate if changes were made. The Creative Commons Public Domain Dedication waiver (http://creativecommons.org/publicdomain/zero/1.0/) applies to the data made available in this article, unless otherwise stated. 


\section{Background}

Clinical risk assessment and decision support tools built into the electronic health record (EHR) are becoming more common. To be successful, these tools need to be user friendly, require minimal data input, apply to broad clinic populations, and integrate seamlessly into clinic work flow [1]. We have been developing a preoperative risk assessment and decision support tool, the Surgical Risk Preoperative Assessment System (SURPAS), for surgical patients at the University of Colorado Hospital that has these desired features and is based on the large database from the American College of Surgeons' National Surgical Quality Improvement Program Participant Use File (ACS NSQIP PUF). This development has involved an iterative process including initial prediction model development [2-4]; development of a prototype tool integrated into the local EHR; focus groups of patients, surgeons, and administrators to review the prototype [5]; pilot testing; and then refinements based upon the focus groups and pilot testing.

In the initial modeling, we found that the 18 30-day postoperative complications collected in the ACS NSQIP could be statistically grouped into six clusters of complications: infectious; pulmonary; cardiac/bleeding; venous thromboembolic; renal; and neurological [2]. Along with 30-day mortality and overall morbidity, this resulted in eight important postoperative adverse outcomes being predicted by the SURPAS tool. We also found that eight preoperative variables could predict risk of these eight adverse outcomes almost as well as using models including up to 40 preoperative ACS NSQIP variables, across the nine surgical subspecialties represented in the ACS NSQIP database. These eight preoperative variables included four patient factors (age, American Society of Anesthesiology physical status classification (ASA class), functional health status prior to surgery (FHS), and presence of systemic sepsis within $48 \mathrm{~h}$ of surgery) and four operative factors (operation complexity as measured by work Relative Value Unit (wRVU), in- or outpatient operation, primary surgeon specialty, and whether or not the operation was an emergency).

In integrating the SURPAS tool into the local EHR (Epic Systems, Verona, WI), we found that only two of the eight SURPAS preoperative predictor variables could be reliably obtained from the EHR at the time of the patient's preoperative encounter-patient age and specialty of the primary surgeon. The other six predictor variables need to be entered by the surgical team.

The focus groups of patients, surgeons, and administrators expressed a number of concerns and/or recommendations for refinements to the prototype SURPAS tool: [5]

1. Related to the eight preoperative predictor variables, preliminary work in integration of
SURPAS into the local EHR suggested that systemic sepsis within $48 \mathrm{~h}$ of surgery would be a difficult variable to assess at the preoperative encounter;

2. Also related to the preoperative variables, there was concern about the adequacy of the wRVU of the primary operation accounting for the complexity of the operation, particularly in operations involving multiple Current Procedural Terminology (CPT) codes;

3. Although a factor analysis of the postoperative complications suggested six clusters of complications, some of the complications that were clustered together are likely addressed by different processes of care; therefore, some should be made distinct postoperative adverse outcomes (e.g., cardiac complications and bleeding were separated; surgical site infections and urinary tract infections (UTIs) were separated, etc.);

4. Hospitalizations causing the patient to spend time away from home and family were identified by our patient partners as important patient concerns; therefore, the risk of unplanned re-hospitalization following surgery should be included as an adverse postoperative outcome;

5. The SURPAS tool should provide documentation of the risk information and discussion with the patient and family in the patient's medical record;

6. The SURPAS tool should provide risk information to the patients and their families in a printed and easily understood format to help them understand and remember details of the informed consent process;

7. The SURPAS tool should provide answers to "frequently asked questions" (FAQs) to facilitate the implementation of the tool and to foster collaborative discussions with patients; and

8. One patient who experienced debilitating depression after his operation suggested incorporating a risk for adverse psychological or cognitive effects postoperatively.

Some of these concerns/recommendations needed to be addressed through further analysis of the ACS NSQIP database (Items \#1, 2, 3, 4); some could be addressed by adding features to the SURPAS tool (Items \#5, 6, 7); and another required the collection of additional outcomes (Item \#8). The purpose of this paper is to report on the further statistical analyses and features added to the SURPAS tool in response to these concerns and recommendations. Our hypotheses were that: (1) Elimination of the preoperative sepsis variable would have negligible effect on fit of the SURPAS predictive models; (2) A predictor variable representing $\mathrm{CPT}$-specific event rate would be an important predictor variable to add to the model in place 
of preoperative sepsis; (3) A predictor variable indicating multiple CPT codes for complex operations would not significantly add to model fit; and (4) Additional adverse outcomes of unplanned readmission, UTI, and bleeding would be successfully predicted by the refined eight SURPAS predictor variables.

\section{Methods}

\section{Study design}

This study was a retrospective, observational study using the 2005-2015 American College of Surgeons National Surgical Quality Improvement Program Participant Use File (ACS-NSQIP PUF). The database includes a systematic sample of major operations performed at $>700$ participating hospitals in nine surgical specialties (general, orthopedic, gynecology, urologic, neurosurgery, otolaryngology, thoracic, plastic, and vascular surgery). Trained clinical nurse reviewers collect preoperative, operative, and 30-day postoperative adverse outcomes on a systematic sample of patients at their hospitals using a standardized protocol and data definitions. The study was ruled exempt from review by the Colorado Multiple Institutional Review Board (COMIRB) because it involved using completely de-identified data from a national database.

\section{Issue 1}

In the previous SURPAS publications, we found that eight preoperative predictor variables could accurately predict eight different postoperative complications across a broad range of surgical operations [2-4]. The final SURPAS models were developed using standard multiple logistic regression analysis, with the adverse postoperative outcomes as the dependent variables and the eight preoperative predictor variables as the independent variables [4]. To address the issue of dropping preoperative sepsis from the group of eight preoperative predictor variables, we performed similar logistic regression models including only seven preoperative variables (eliminating preoperative sepsis) and compared the c-indexes and Brier scores of the seven-variable models to the original eight-variable models.

We added a predictor variable that we call "CPT-specific event rate." This variable is defined as the event rate of the CPT code of the primary operation for the outcome being modeled, calculated from the ACS NSQIP database for the years 2005-2015 that included over 4.6 million observations from over 700 participating hospitals nationwide. We reasoned that CPT-specific event rate calculated from past operations should be a good predictor of the outcome for future operations of the same type (CPT code). We then compared the c-indexes and Brier scores of the eight-variable models that included "CPT-specific event rate" with the original eightvariable models that included preoperative sepsis.

\section{Issue 2}

To address the issue of operations with multiple CPT codes being more complex than is represented by the wRVU value, we ran nine-variable models adding an indicator variable for operations with multiple CPT codes $(0=$ no, $1=$ yes $)$ and compared the c-indexes and Brier scores for these nine-variable models to the eight-variable models that included CPT-specific event rate to see if performing operations involving multiple CPT codes added to the risk of the patient. We used an indicator variable for multiple CPT codes, rather than using the anticipated number of CPT codes or the actual CPT codes because we reasoned that at the preoperative encounter, the surgeon might know that the operation would involve multiple CPT codes but might not know exactly which ones would be involved or how many.

\section{Issues 3 \& 4}

To address the issues related to the complication clusters and hospitalizations we developed models for a new set of eleven adverse postoperative outcomes that separated bleeding/transfusion from the cardiac outcomes, UTI from the other infection outcomes, and unplanned readmission as an additional adverse postoperative outcome. To validate the new eight-variable models, we randomly split the cases 50:50 into developmental and test datasets, developed the models in the developmental dataset and then tested the developed models in the test dataset and compared c-indexes and Brier scores between the developmental and test samples. We hypothesized that CPT-specific event rate would be one of the more important predictor variables of the eight-variable set of predictors. To assess this, we ran forward selection logistic regression models forcing all eight predictor variables into the models, and compared orders of entry of the variables across the eleven adverse outcomes.

\section{Issue $\mathbf{5}$}

To address the documentation of the risk information and discussion with the patient and family we added a feature to SURPAS that would automatically generate a pre-operative note in the patient's EHR summarizing the patient's individualized preoperative risks compared to the national average for patients undergoing the same operation (Fig. 2), and a note that these data were discussed with the patient.

\section{Issue 6}

To address the issue of providing a graphical display of the patient's risks to the patient and family, we added a feature to SURPAS to allow a pictograph of the individual patient's risk compared to the national average to be printed out to give to the patient at the preoperative encounter (Fig. 3). We gave the focus groups of patients several different examples of how these data could be 
graphically displayed (including a bar graph, pictograph with stick figures, pictograph with boxes, pie graph, sparkplug display, clock graph display, and a table of numerical values) [6-8]. The majority of patients preferred the pictograph for the display of the risks [9].

\section{Issue 7}

To address the issue of providing answers to "frequently asked questions" we developed an "About" section for SURPAS to describe the development of the tool and the underlying methodology (Fig. 4).

\section{Issue 8}

We believed that issues such as long term outcomes and risk for adverse psychological or cognitive effects from the operation were beyond the scope of the current SURPAS development. These will likely involve a different type of data collection and collection longitudinally over time, both before and after the operation. But this focus group comment has prompted us to explore the potential for adding patient-reported outcomes to the SURPAS tool, which will be the subject of future grant applications and publications.

\section{Results}

Additional file 1 Figure S1 presents the STROBE diagram for the development of the analytic database. In the period 2005-2015, there were 4.6 million operations in the ACS NSQIP database. We eliminated $0.6 \%$ of the operations for not being one of the nine surgical specialties targeted by the ACS NSQIP and SURPAS (general, orthopedic, vascular, gynecology, urology, otolaryngology, plastic, thoracic, and neurosurgery), and $0.8 \%$ of the operations for having missing data on key SURPAS predictor variables (functional health status, ASA class, age, emergency operation, or inpatient/outpatient operation). This resulted in 4.54 million operations being included in the analytic file (98.6\% of the total operations). Additional file 1: Table S1 presents the patient characteristics, including the SURPAS predictor variables, and the rates for the eleven postoperative adverse outcomes for these 4.54 million patients.

\section{Issue 1}

In Table 1, we see that elimination of the preoperative sepsis predictor variable had very little effect on the c-indexes and Brier scores for predicting the eleven outcome variables. C-indexes were decreased by 0.001 to 0.013 units, or percent decreases of 0.1 to $1.7 \%$, while Brier scores were increased by 0.0000 to 0.0018 units, or percent increases of 0.0 to $3.9 \%$.

When CPT event rate was substituted for preoperative sepsis in a new eight-variable model, c- indexes were increased by 0.002 to 0.031 units, or percent increases of 0.2 to $4.0 \%$ (Table 2). Brier scores did not change for five of the eleven adverse postoperative outcomes, were reduced for four additional outcomes by 0.0001 to 0.0024 units (percent reductions of $-0.2 \%$ to $-2.8 \%$ ), and were increased for only two outcomes by 0.0001 units (percent increase of 1.0\%) and 0.0005 units (percent increase of $2.2 \%$ ). The second column in Table 2 gives the c-indexes for the SURPAS prediction models that are in current use. The c-index is above 0.90 for one outcome (mortality, 0.928), between 0.80 and 0.89 for seven outcomes (0.893 for pulmonary, 0.875 for bleeding/transfusion, 0.871 for cardiac, 0.863 for renal, 0.840 for stroke, 0.823 for overall morbidity, and 0.805 for infection), and between 0.70 and 0.79 for three outcomes ( 0.788 for VTE, 0.776 for UTI, and 0.723 for unplanned readmission).

Table 1 Comparison of Discrimination and Calibration of the Full Eight Variable SURPAS Model with Models without Systemic Sepsis $^{a}$

\begin{tabular}{|c|c|c|c|c|c|c|}
\hline \multirow[t]{2}{*}{ Adverse outcome } & \multicolumn{3}{|l|}{ C-index } & \multicolumn{3}{|l|}{ Brier Score } \\
\hline & Full Model & Full Model with No Systemic Sepsis & Difference & Full Model & Full Model with No Systemic Sepsis & Difference \\
\hline 30-day mortality & 0.926 & 0.922 & -0.004 & 0.0102 & 0.0103 & 0.0001 \\
\hline Morbidity & 0.801 & 0.793 & -0.008 & 0.0904 & 0.0922 & 0.0018 \\
\hline Readmission & 0.699 & 0.698 & -0.001 & 0.0488 & 0.0489 & 0.0001 \\
\hline Infection & 0.774 & 0.761 & -0.013 & 0.0445 & 0.0450 & 0.0005 \\
\hline Bleeding/Transfusion & 0.850 & 0.846 & -0.004 & 0.0419 & 0.0421 & 0.0002 \\
\hline Pulmonary & 0.890 & 0.882 & -0.008 & 0.0228 & 0.0237 & 0.0009 \\
\hline UTI & 0.750 & 0.749 & -0.001 & 0.0145 & 0.0145 & 0.0000 \\
\hline VTE & 0.765 & 0.760 & -0.005 & 0.0086 & 0.0086 & 0.0000 \\
\hline Cardiac & 0.869 & 0.866 & -0.003 & 0.0062 & 0.0062 & 0.0000 \\
\hline Renal & 0.859 & 0.853 & -0.006 & 0.0059 & 0.0059 & 0.0000 \\
\hline Neurologic & 0.829 & 0.828 & -0.001 & 0.0021 & 0.0021 & 0.0000 \\
\hline
\end{tabular}

Abbreviations: SURPAS Surgical Preoperative Risk Assessment System, UTI Urinary tract infection, VTE Venous thromboembolism 
Table 2 Comparison of Discrimination and Calibration of the Full Eight Variable SURPAS Model with Models without Systemic Sepsis but with the Addition of CPT Specific Event Rates ${ }^{a}$

\begin{tabular}{|c|c|c|c|c|c|c|}
\hline \multirow[t]{2}{*}{ Adverse outcome } & \multicolumn{3}{|l|}{ C-index } & \multicolumn{3}{|l|}{ Brier Score } \\
\hline & Full Model & Full Model with CPT Event Rate & Difference & Full Model & Full Model with CPT Event Rate & Difference \\
\hline 30-day mortality & 0.926 & 0.928 & 0.002 & 0.0102 & 0.0103 & 0.0001 \\
\hline Morbidity & 0.801 & 0.823 & 0.022 & 0.0904 & 0.0880 & -0.0024 \\
\hline Readmission & 0.699 & 0.723 & 0.024 & 0.0488 & 0.0487 & -0.0001 \\
\hline Infection & 0.774 & 0.805 & 0.031 & 0.0445 & 0.0442 & -0.0003 \\
\hline Bleeding/Transfusion & 0.850 & 0.875 & 0.025 & 0.0419 & 0.0408 & -0.0011 \\
\hline Pulmonary & 0.890 & 0.893 & 0.003 & 0.0228 & 0.0233 & 0.0005 \\
\hline UTI & 0.750 & 0.776 & 0.026 & 0.0145 & 0.0145 & 0.0000 \\
\hline VTE & 0.765 & 0.788 & 0.023 & 0.0086 & 0.0086 & 0.0000 \\
\hline Cardiac & 0.869 & 0.871 & 0.002 & 0.0062 & 0.0062 & 0.0000 \\
\hline Renal & 0.859 & 0.863 & 0.004 & 0.0059 & 0.0059 & 0.0000 \\
\hline Neurologic & 0.829 & 0.840 & 0.011 & 0.0021 & 0.0021 & 0.0000 \\
\hline
\end{tabular}

Abbreviations: SURPAS Surgical Preoperative Risk Assessment System, UTI Urinary tract infection, VTE Venous thromboembolism

\section{Issue 2}

To examine whether adding an indicator variable for multiple CPT codes would improve the SURPAS prediction models, we compared nine-variable models (with the addition of an indicator variable for multiple CPT codes) to our eight-variable models (Table 3). The addition of the indicator variable minimally increased the c-indexes by 0.000 to 0.002 units $(0.0$ to $0.2 \%)$, and minimally decreased the Brier scores by 0.0000 to 0.0004 units (decreases of 0.0 to $0.5 \%$ ).

\section{Issues 3 \& 4}

Models were developed for a revised set of eleven adverse postoperative outcomes that separated bleeding/ transfusion from the cardiac outcomes, UTI from the other infection outcomes, and added a predictive model for unplanned readmission. Table 4 presents the internal validation statistics for the eight-variable SURPAS prediction models, not including preoperative sepsis and including CPT-specific event rate, for the eleven SURPAS adverse postoperative outcomes. The table gives the order of entry of the eight SURPAS prediction variables across the eleven different postoperative adverse outcomes and the average order of entry. CPT-specific event rate was the first variable to enter the models for nine of the eleven adverse postoperative outcomes (all but the models for 30-day mortality and cardiac complications in which ASA class was the first variable to enter). Comparing the c-indexes and Brier scores of the prediction models

Table 3 Comparison of Discrimination and Calibration of the SURPAS without Systemic Sepsis but with the Addition of CPT Specific Event Rates with Models adding an Indicator for Multiple CPTs ${ }^{\text {a }}$

\begin{tabular}{|c|c|c|c|c|c|c|}
\hline \multirow[t]{2}{*}{ Adverse outcome } & \multicolumn{3}{|l|}{ C-index } & \multicolumn{3}{|l|}{ Brier Score } \\
\hline & $\begin{array}{l}\text { Full Model with } \\
\text { CPT Event Rate }\end{array}$ & $\begin{array}{l}\text { Same Model plus } \\
\text { Multiple CPT Indicator }\end{array}$ & Difference & $\begin{array}{l}\text { Full Model with } \\
\text { CPT Event Rate }\end{array}$ & $\begin{array}{l}\text { Same Model plus } \\
\text { Multiple CPT Indicator }\end{array}$ & Difference \\
\hline 30-day mortality & 0.928 & 0.929 & 0.001 & 0.0103 & 0.0103 & 0.0000 \\
\hline Morbidity & 0.823 & 0.824 & 0.001 & 0.0880 & 0.0876 & -0.0004 \\
\hline Readmission & 0.723 & 0.723 & 0.000 & 0.0487 & 0.0487 & 0.0000 \\
\hline Infection & 0.805 & 0.806 & 0.001 & 0.0442 & 0.0441 & -0.0001 \\
\hline Bleeding/Transfusion & 0.875 & 0.877 & 0.002 & 0.0408 & 0.0406 & -0.0002 \\
\hline Pulmonary & 0.893 & 0.894 & 0.001 & 0.0233 & 0.0232 & -0.0001 \\
\hline UTI & 0.776 & 0.777 & 0.001 & 0.0145 & 0.0145 & 0.0000 \\
\hline VTE & 0.788 & 0.789 & 0.001 & 0.0086 & 0.0086 & 0.0000 \\
\hline Cardiac & 0.871 & 0.871 & 0.000 & 0.0062 & 0.0062 & 0.0000 \\
\hline Renal & 0.863 & 0.865 & 0.002 & 0.0059 & 0.0059 & 0.0000 \\
\hline Neurologic & 0.840 & 0.84 & 0.000 & 0.0021 & 0.0021 & 0.0000 \\
\hline
\end{tabular}

${ }^{a}$ Abbreviations: SURPAS Surgical Preoperative Risk Assessment System, UTI Urinary tract infection, VTE Venous thromboembolism 
Table 4 Order of Entry of Predictor Variables in the Final SURPAS Models and C-indexes and Brier Scores for the Development and Test Datasets in the Internal Validation Study

\begin{tabular}{|c|c|c|c|c|c|c|c|c|c|c|c|c|}
\hline Characteristics $^{a}$ & $\begin{array}{l}\text { 30-day } \\
\text { Mortality }\end{array}$ & Morbidity & $\begin{array}{l}\text { Unplanned } \\
\text { Readmission }\end{array}$ & Respiratory & Infection & UTI & VTE & Cardiac & Bleeding & Renal & Stroke & $\begin{array}{l}\text { Average } \\
\text { Rank }\end{array}$ \\
\hline CPT-specific event rate & 2 & 1 & 1 & 1 & 1 & 1 & 1 & 2 & 1 & 1 & 1 & 1.2 \\
\hline ASA class & 1 & 2 & 2 & 2 & 3 & 2 & 3 & 1 & 2 & 2 & 2 & 2.0 \\
\hline Inpatient/outpatient & 6 & 3 & 3 & 3 & 2 & 3 & 2 & 4 & 3 & 3 & 4 & 3.3 \\
\hline Primary surgeon specialty & 7 & 4 & 4 & 6 & 4 & 4 & 6 & 6 & 4 & 4 & 5 & 4.9 \\
\hline Age (years) & 4 & 7 & 7 & 7 & 6 & 5 & 4 & 3 & 5 & 7 & 3 & 5.3 \\
\hline Functional health status & 3 & 5 & 5 & 4 & 7 & 6 & 5 & 7 & 8 & 6 & 6 & 5.6 \\
\hline Emergency operation & 5 & 6 & 8 & 5 & 5 & 8 & 7 & 5 & 7 & 5 & 7 & 6.2 \\
\hline Work relative value unit & 8 & 8 & 6 & 8 & 8 & 7 & 8 & 8 & 6 & 8 & 8 & 7.5 \\
\hline Model c-index & 0.928 & 0.823 & 0.724 & 0.893 & 0.805 & 0.777 & 0.788 & 0.871 & 0.875 & 0.863 & 0.839 & \\
\hline Model Brier score & 0.0103 & 0.0879 & 0.0485 & 0.0234 & 0.0438 & 0.0145 & 0.0086 & 0.0062 & 0.0406 & 0.0060 & 0.0021 & \\
\hline Validation c-index & 0.931 & 0.823 & 0.724 & 0.893 & 0.805 & 0.776 & 0.790 & 0.877 & 0.876 & 0.870 & 0.875 & \\
\hline Validation Brier score & 0.0102 & 0.0880 & 0.0483 & 0.0232 & 0.0437 & 0.0144 & 0.0086 & 0.0063 & 0.0407 & 0.0059 & 0.0020 & \\
\hline
\end{tabular}

${ }^{a}$ Abbreviations: ASA class American Society of Anesthesiology physical status classification, CPT Current Procedural Terminology, SURPAS Surgical Preoperative Risk Assessment System, UTI Urinary tract infection, VTE Venous thromboembolism

in the developmental and test datasets, only one of the eleven c- indexes (for UTI) showed the expected decline going from the developmental to the test dataset and that decline was only 0.001 unit, and all changes in Brier scores from the development to the test dataset were within 0.002 units, indicating excellent internal validation.
Issues 5, 6, and 7

Figure 1 shows the SURPAS screen seen by the surgeon at the preoperative visit for a patient undergoing a pancreatojejunostomy. The data input values are on the left: name and/or CPT code of the primary operation; age of the patient (automatically populated from the EHR); functional health status; ASA class; inpatient/outpatient

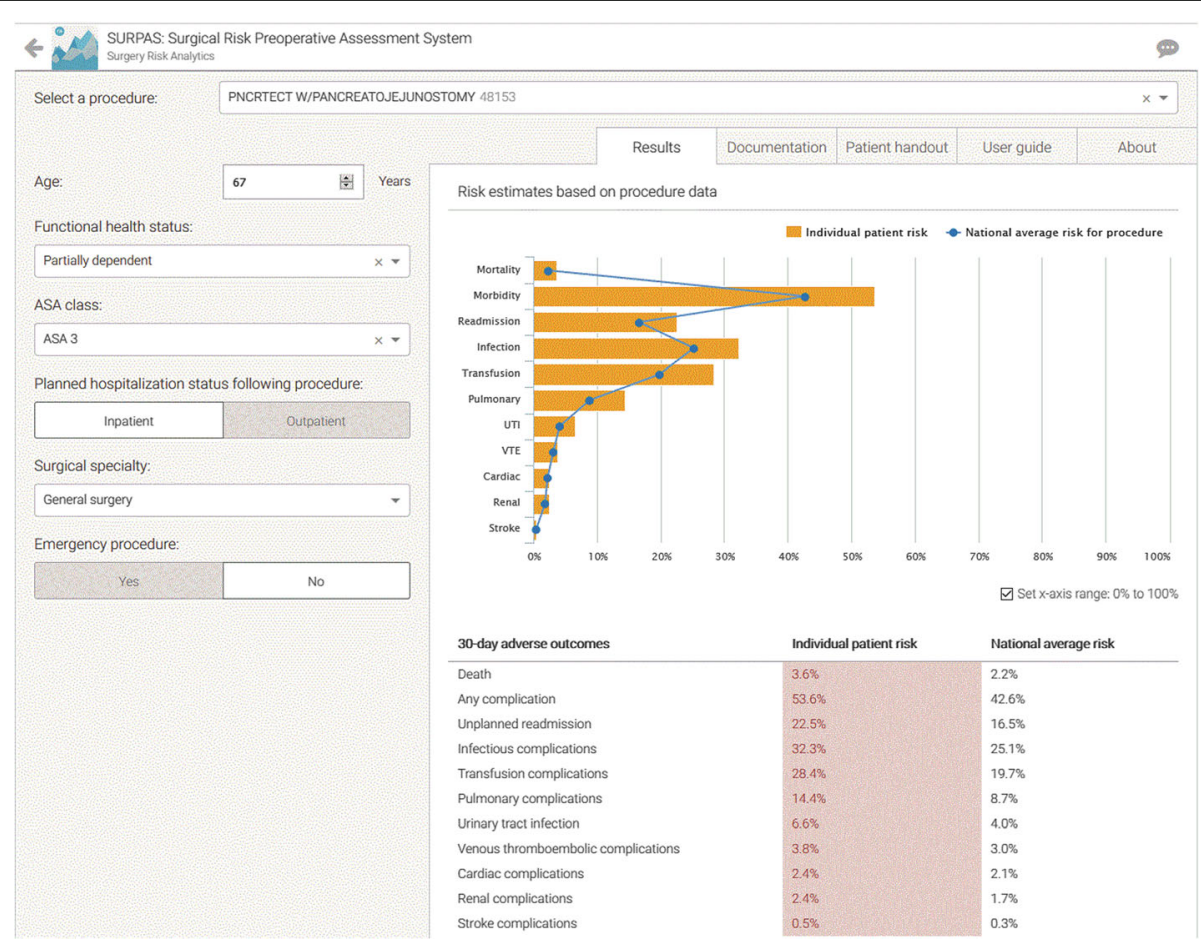

Fig. 1 SURPAS Input and Output Screen 
procedure; surgical specialty of the primary surgeon (automatically populated from the EHR); and whether the surgical procedure is done on an emergency basis. Once the CPT code is specified, a table look-up can obtain values for wRVU and CPT-specific event rates. Individualized patient risks for the eleven postoperative adverse outcomes (yellow bars) compared to national averages (blue dots) are given on the right both graphically and in table form. A preoperative note is automatically created for import into the patient's EHR (Fig. 2). A pictograph of the patient risks compared to national averages (Fig. 3-showing abbreviated pictograph for mortality and overall morbidity) for all eleven of the postoperative adverse outcomes can be printed out for the patient to keep. An "About" section (Fig. 4) can be referenced by the provider to explain the SURPAS tool, and a "User guide" demonstrates to providers how to use the SURPAS tool.

\section{Issue 8}

Long term functional outcomes are beyond the scope of SURPAS due to lack of long term and functional outcomes in the ACS NSQIP PUF. However, these may possibly be available through incorporation of patient reported outcomes such as those measured by the Patient Reported Outcomes Measurement Information System, and piloted by the ACS NSQIP in 2018 (personal communication from Dr. Jason Lyu) [10].

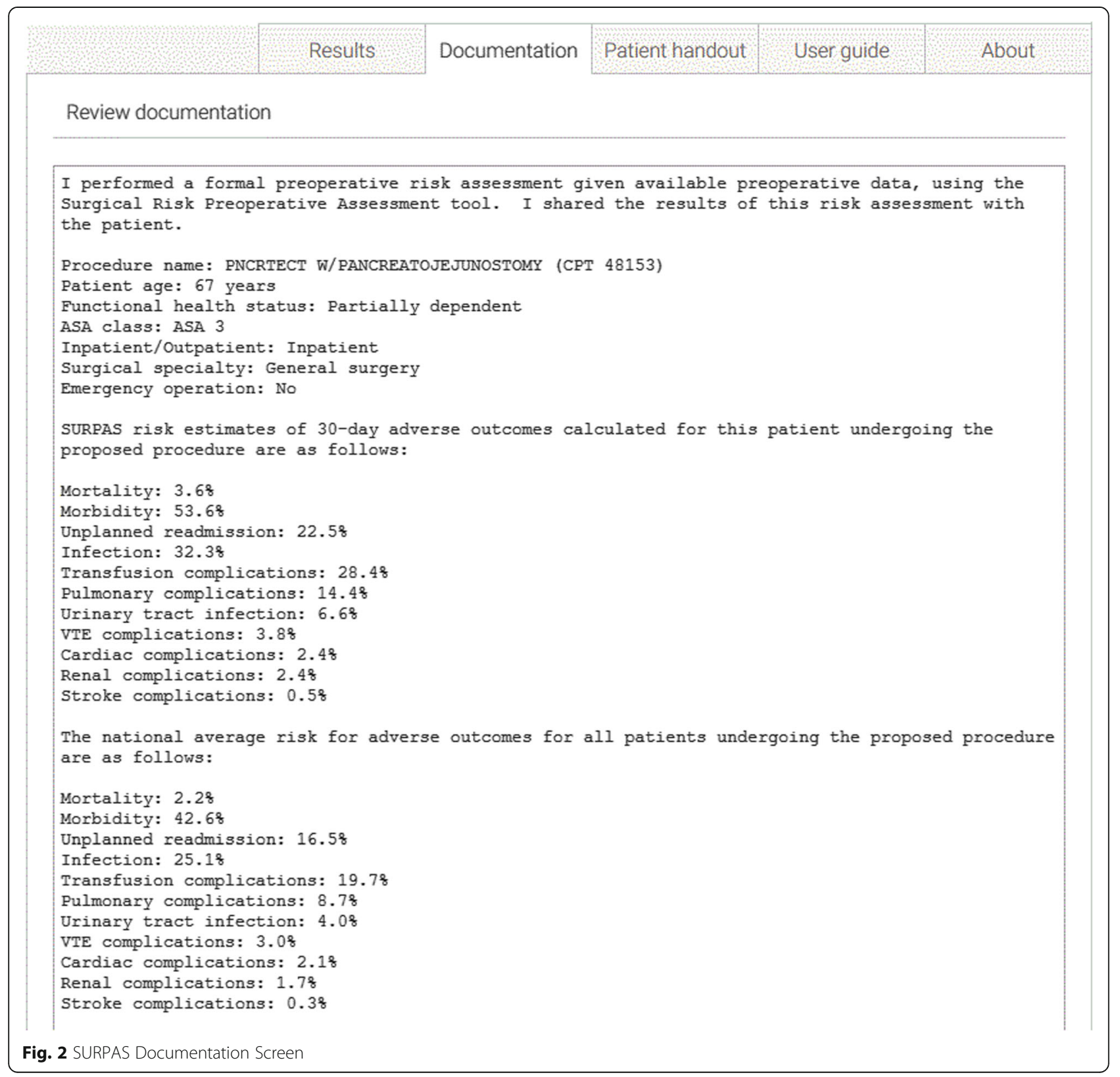




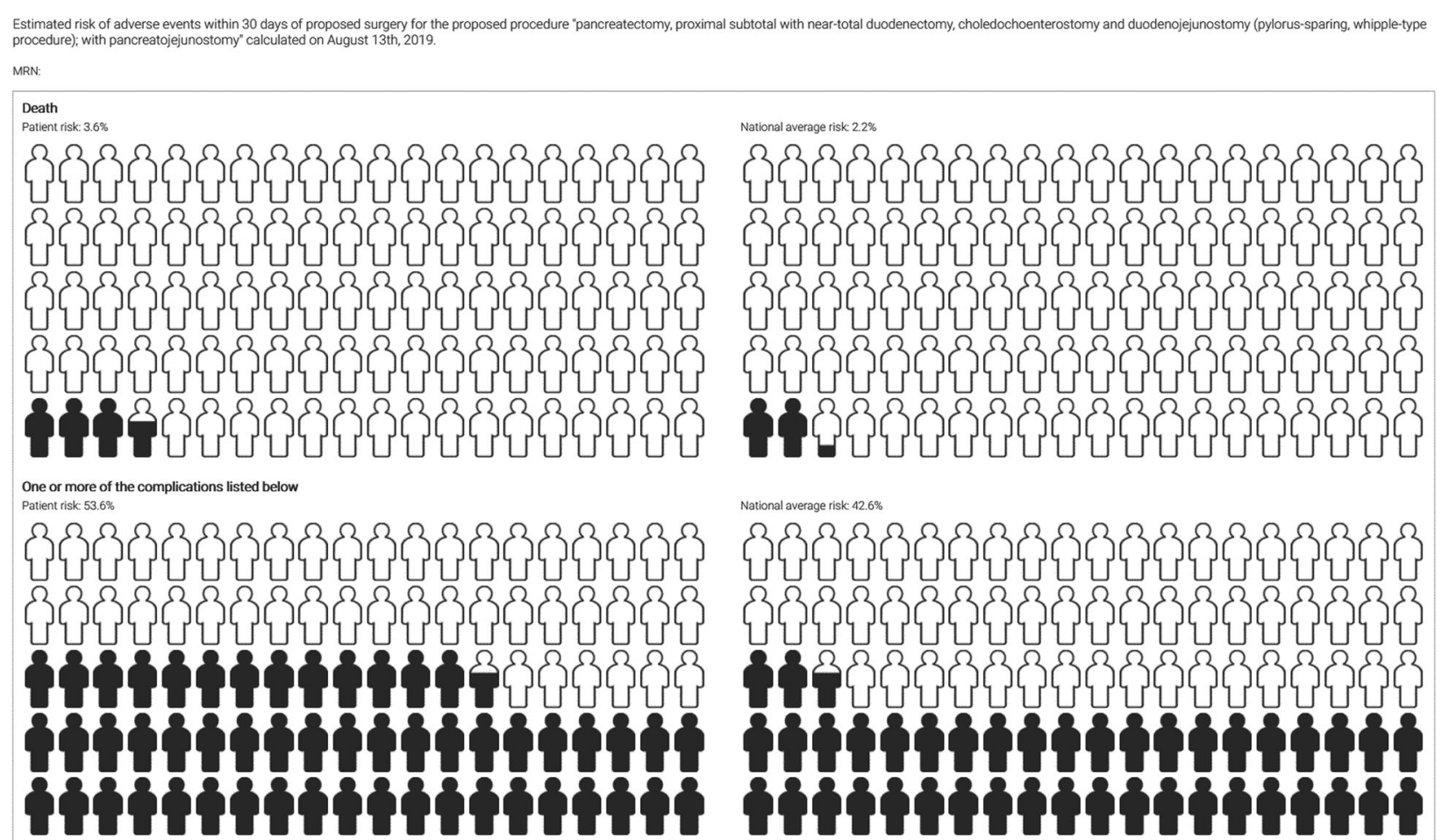

Fig. 3 SURPAS Patient handout, abbreviated

\section{Discussion}

In this paper, we settled on the revised SURPAS prediction models currently in use in the implementation and dissemination of SURPAS at the UCHealth hospitals. Based on feedback from our focus groups of patients, surgeons, and administrators we added three postoperative adverse outcomes to the original eight SURPAS outcomes-bleeding, UTI, and unplanned readmission. We showed that the 11 SURPAS prediction models have good to excellent $c$-indexes and Brier scores. Preoperative sepsis was replaced as a predictor variable because it was difficult ascertaining this variable preoperatively from the EHR and is unlikely to be present in elective surgical patients, and in its place we added CPT-specific event rate calculated from the large, national ACS NSQIP database of 4.54 million operations. Dropping the preoperative sepsis variable did not have a significant effect on the c-indexes and Brier scores for the prediction models, but adding CPT-specific event rate did significantly improve these measures for several of the prediction models. Another advantage of the CPT-specific event rate is that it does not require additional data input - once the provider enters the name or CPT code of the operation, internal look-ups obtain the values for wRVU and CPT-specific event rates. We reasoned that the simple addition of an indicator for multiple concurrent procedures would be easier for the provider to anticipate, than actually identifying and listing the additional procedures in the SURPAS tool while minimizing intrusion into the compressed preoperative encounter. We showed that adding this did not significantly change the c-indexes and Brier scores for the models, implying that our models do tend to account for the complexity of operations with multiple CPT codes. This might happen if the multiple CPT codes tend to often occur together so that the risk of the complex operation is already built into the CPT code of the primary operation through the CPT-specific event rate. The internal validation study showed that there was very little loss of discrimination and calibration in going from the developmental to the test dataset.

We believe that adding the features of the automatically generated preoperative note, the pictograph of the individual patient risks compared to the national averages, which can be printed out and handed to the patient at the preoperative visit, and the "About" section and "User guide" in the SURPAS tool will enhance the utility of SURPAS to patients and providers. We believe the ability to provide a patient with their risk of postoperative adverse event, review it with them in a meaningful manner, and then provide a hard copy of this information so that they may further consider the risks after leaving the encounter and more clearly 
The Surgical Risk Preoperative Assessment System (SURPAS) is a risk assessment tool intended for use by patient care providers to estimate individual patient risk of complications after a surgical procedure. This quantitative preoperative risk assessment is based upon an individual patient's values for eight preoperatively available risk factors. These eight preoperatively available risk factors carry nearly all of the prognostic information available in 40 preoperatively available risk factors contained in the American College of Surgeons (ACS) National Surgical Quality Improvement Program (NSQIP) database because many risk factors carry redundant prognostic information. The SURPAS tool contains equations based on analyses of over 4 million surgical procedures contained in the ACS NSQIP database (2005-2015). This risk assessment is only an estimate and not exact; individual patient risks may be greater or lower. Providers should use the guidance of the output of SURPAS to educate patients and further optimize their care to decrease adverse postoperative outcomes.

For procedures performed with a frequency of less than 100 times within the source data set, surgeon specialty-specific event rates are used in the prediction models rather than CPT-specific event rates.

SURPAS estimates the probability of 30-day postoperative adverse events for eleven different outcomes, including mortality, readmission, 8 different specific types of postoperative complications (infection, transfusion, pulmonary, urinary tract infection, venous thromboembolism, cardiac, renal, and stroke), and overall morbidity ( 1 or more of the 8 specific types of complications). The prediction models using the 8 SURPAS predictor variables were developed independently, using logistic regression analysis. It is possible, but rare (estimated to be $<2 \%$ of cases), that the probability of an individual complication is larger than the probability of overall morbidity. We believe that this happens because the models were developed independently and there is slight variation in the quality of the models' predictions of the individual outcomes. We estimate that in $87 \%$ of these anomalous cases the percent increase in the probability for the specific complication compared to overall morbidity will be $\leq 20 \%$.

The development of the models is detailed in the following publications (* indicates co-first authors):

- Meguid, R.A.*, Bronsert, M.R.*, Juarez-Colunga, E., Hammermeister, K.E., and Henderson, W.G.: Surgical Risk Preoperative Assessment System (SURPAS): I. Parsimonious, Clinically Meaningful Groups of Postoperative Complications by Factor Analysis. the Annals of Surgery, June; 263(6):1042-8, 2016. PMID 26954897

- Meguid, R.A.*, Bronsert, M.R.*, Juarez-Colunga, E.*, Hammermeister, K.E., and Henderson, W.G.: Surgical Risk Preoperative Assessment System (SURPAS): II. Parsimonious Risk Models for Postoperative Adverse Outcomes Addressing need for Laboratory Variables and Surgeon Specialty-Specific Models. the Annals of Surgery, July; 264(1):10-22, 2016. PMID 26945154

- Meguid, R.A., Juarez-Colunga, E., Bronsert, M.R., Hammermeister, K.E. and Henderson, W.G.: Surgical Risk Preoperative Assessment System (SURPAS): III. Preoperative Prediction of Adverse Outcomes with Eight Predictor Variables. the Annals of Surgery, July; 264(1):23-31, 2016. PMID 26928465

American College of Surgeons National Surgical Quality Improvement Program:

- https://www.facs.org/quality-programs/acs-nsqip

- Hall BL, Richards K, Ingraham A et al. New approaches to the National Surgical Quality Improvement Program: the American College of Surgeons experience. The American Journal of Surgery 2009; 198:S56-S62.

Funding for the development of SURPAS:

- Department of Surgery, University of Colorado School of Medicine

- Agency for Healthcare Research and Quality grant 1R21HS024124-01

Fig. 4 SURPAS About section

relate them to their family will improve their engagement in shared decision making [11].

The mention of cognitive effects of the operation by one patient in the focus groups prompted us to explore the possibility of adding patient reported outcomes to SURPAS. Adverse effects from operations on traits such as cognition, pain, physical functioning, or performance of activities of daily living require longitudinal measurements before and after the operation reported by the patient. We considered this work to be beyond the scope of the first version of the SURPAS tool, but we plan to explore this further in the future.

We have compared the SURPAS models to the American College of Surgeons Surgical Risk Calculator, finding good correlation for overall morbidity [12]. Although we performed an internal validation of the new SURPAS predictive models in this paper and found that they validated well, the SURPAS models also need to be validated externally in future prospective studies.

We view the development, implementation, and dissemination of the SURPAS tool at UCHealth as a long-term project. In addition to exploring patient reported outcomes, future research involving SURPAS will need to address issues such as how to define patients at "high risk" for adverse outcomes, and identifying and testing processes of care that might mitigate their risk and consequently prevent postoperative complications in patients.

\section{Conclusion}

Based upon focus groups of patients, surgeons, and administrators we made refinements to SURPAS. These were successful in improving the accuracy of the models, 
while reducing manual entry to five of the eight variables. Adding a predictor variable to indicate a complex operation with multiple current procedure codes did not improve the accuracy of the models, therefore SURPAS will use of the primary procedure (CPT code with the greatest wRVU). We developed graphical displays of risk for providers and patients - a take-home handout for patients and an automated documentation of risk and the discussion in the electronic health record. These improvements should facilitate easier use and implementation of SURPAS. Future prospective external validation studies of the SURPAS models are needed.

\section{Additional file}

Additional file 1: Table S1. Patient Characteristics and Adverse Outcome Rates for Study Cohort. Figure S1. Strengthening the Reporting of Observation studies in Epidemiology (STROBE) diagram. (DOCX $31 \mathrm{~kb})$

\section{Abbreviations}

ACS NSQIP PUF: American College of Surgeons' National Surgical Quality Improvement Program Participant Use File; AHRQ: Agency for Healthcare Research and Quality; ASA class: American Society of Anesthesiology physical status classification; COMIRB: Colorado Multiple Institutional Review Board; CPT: Current Procedural Terminology; EHR: Electronic health record; FAQs: Frequently asked questions; FHS: Functional health status prior to surgery; SURPAS: Surgical Risk Preoperative Assessment System; UTI: Urinary tract infection; wRVU: Work Relative Value Unit

\section{Acknowledgements}

Not applicable.

\section{Authors' contributions}

RAM, ALK, KEH, WGH, and MRB guided the overall project. RAM, KEH, WGH, and $M R B$ conducted the quantitative data analyses that provided the background data and the creation of SURPAS. WGH and RAM were major contributors in writing the manuscript with support from KEH, WGH, and ALK. All authors read and approved the final manuscript.

\section{Funding}

This study was supported by a grant from the Agency for Healthcare Research and Quality (AHRQ) 1R21HS024124-02 grant. The Agency for Healthcare Research and Quality had no role in the design of the study and collection, analysis, and interpretation of data or in writing the manuscript.

\section{Availability of data and materials}

The datasets used and analyzed during the current study are the ACS NSQIP PUF. These data are the property of the American College of Surgeons, and are freely available to faculty and staff at institutions participating in the ACS NSQIP.

\section{Ethics approval and consent to participate}

Not applicable.

\section{Consent for publication}

Not applicable.

\section{Competing interests}

The authors report no conflicts and do not derive any financial gain from SURPAS.

\section{Author details}

'Surgical Outcomes and Applied Research program, Department of Surgery, University of Colorado School of Medicine, Aurora, CO, USA. ${ }^{2}$ Adult and Child Center for Health Outcomes Research and Delivery Science, University of
Colorado School of Medicine, Aurora, CO, USA. ${ }^{3}$ Department of Biostatistics and Informatics, Colorado School of Public Health, Aurora, CO, USA. ${ }^{4}$ Division of Cardiology, Department of Medicine, University of Colorado School of Medicine, Aurora, CO, USA. ${ }^{5}$ VA Eastern Colorado Health Care System, Department of Veterans Affairs Medical Center, Aurora, CO, USA. ${ }^{6}$ Division of Cardiothoracic Surgery, Department of Surgery, University of Colorado Denver | Anschutz Medical Campus, 12631 E. 17th Avenue, C-310, Aurora, CO 80045, USA

Received: 2 April 2019 Accepted: 5 August 2019

Published online: 20 August 2019

\section{References}

1. Hammermeister KE, Henderson WG, Bronsert MR, Juarez-Colunga E, Meguid RA. Bringing quantitative risk assessment closer to the patient and surgeon: a novel approach to improve outcomes. Ann Surg. 2016;263:1039-41.

2. Meguid RA, Bronsert MR, Juarez-Colunga E, Hammermeister KE, Henderson WG. Surgical risk preoperative assessment system (SURPAS): I. parsimonious, clinically meaningful groups of postoperative complications by factor analysis. Ann Surg. 2016;263(6):1042-8.

3. Meguid RA, Bronsert MR, Juarez-Colunga E, Hammermeister KE, Henderson WG. Surgical risk preoperative assessment system (SURPAS): II. Parsimonious risk models for postoperative adverse outcomes addressing need for laboratory variables and surgeon specialty-specific models. Ann Surg. 2016; 264(1):10-22.

4. Meguid RA, Bronsert MR, Juarez-Colunga E, Hammermeister KE, Henderson WG. Surgical risk preoperative assessment system (SURPAS): III. Accurate preoperative prediction of 8 adverse outcomes using 8 predictor variables. Ann Surg. 2016;264(1):23-31.

5. Lambert-Kerzner A, Ford KL, Hammermeister KE, Henderson WG, Bronsert MR, Meguid RA. Assessment of attitudes towards future implementation of the "surgical risk preoperative assessment system" (SURPAS) tool: a pilot survey among patients, surgeons, and hospital administrators. Patient Saf Surg. 2018;12:12.

6. Hawley ST, Zikmund-Fisher B, Ubel P, Jancovic A, Lucas T, Fagerlin A. The impact of the format of graphical presentation on health-related knowledge and treatment choices. Patient Educ Couns. 2008;73(3):448-55.

7. Zikmund-Fisher BJ, Witteman HO, Dickson M, et al. Blocks, ovals, or people? Icon type affects risk perceptions and recall of pictographs. MedDecisMaking. 2014;34(4):443-53.

8. Fagerlin A, Zikmund-Fisher BJ, Ubel PA. Helping patients decide: ten steps to better risk communication. JNat|Cancer Inst. 2011;103(19):1436-43.

9. Lambert-Kerzner A, Ford KL, Hammermeister KE, Henderson WG, Bronsert MR, Meguid RA. Assessment of attitudes towards future implementation of the surgical risk preoperative assessment system (SURPAS) tool: a qualitative study. Patient Safety in Surgery. 2018; In Press.

10. Northwest University. Patient-Reported Outcomes Measurement Information System. 2019;9:2019. Accessed January. http://www.healthmeasures.net/ explore-measurement-systems/promis.

11. Kruser JM, Taylor $\amalg$, Campbell TC, et al. "Best case/worst case": training surgeons to use a novel communication tool for high-risk acute surgical problems. J Pain Symptom Manag 2017;53(4):711-719 e715.

12. Khaneki S, Bronsert MR, Henderson WG, et al. Comparison of accuracy of prediction of postoperative mortality and morbidity between a new, parsimonious risk calculator (SURPAS) and the ACS surgical risk calculator. American Journal of Surgery, in press, July. 2019;27.

\section{Publisher's Note}

Springer Nature remains neutral with regard to jurisdictional claims in published maps and institutional affiliations. 